\title{
Ultrafast thermoelectric properties of gold under conditions of strong electron-phonon nonequilibrium
}

\author{
Patrick E. Hopkins, ${ }^{1, a)}$ Matthew L. Bauer, ${ }^{2}$ John C. Duda, ${ }^{2}$ Justin L. Smoyer, ${ }^{2}$ \\ Timothy S. English, ${ }^{2}$ Pamela M. Norris, ${ }^{2}$ Thomas E. Beechem, ${ }^{1}$ and Derek A. Stewart ${ }^{3}$ \\ ${ }^{1}$ Sandia National Laboratories, Albuquerque, New Mexico 87185-0346, USA \\ ${ }^{2}$ Department of Mechanical and Aerospace Engineering, University of Virginia, Charlottesville, Virginia \\ 22904-4746, USA \\ ${ }^{3}$ Cornell Nanoscale Science and Technology Facility, Cornell University, Ithaca, New York 14853-2700, \\ USA
}

(Received 17 August 2010; accepted 2 October 2010; published online 23 November 2010)

\begin{abstract}
The electronic scattering rates in metals after ultrashort pulsed laser heating can be drastically different than those predicted from free electron theory. The large electron temperature achieved after ultrashort pulsed absorption and subsequent thermalization can lead to excitation of subconduction band thermal excitations of electron orbitals far below the Fermi energy. In the case of noble metals, which all have a characteristic flat $d$-band several electron volts well below the Fermi energy, the onset of $d$-band excitations has been shown to increase electron-phonon scattering rates by an order of magnitude. In this paper, we investigate the effects of these large electronic thermal excitations on the ultrafast thermoelectric transport properties of gold, a characteristic noble metal. Under conditions of strong electron-phonon nonequilibrium (relatively high electron temperatures and relatively low lattice temperatures, $T_{e} \gg T_{L}$ ), we find that the Wiedemann-Franz law breaks down and the Seebeck coefficient is massively enhanced. Although we perform representative calculations for $\mathrm{Au}$, these results are expected to be similar for the other noble metals ( $\mathrm{Ag}$ and $\mathrm{Cu}$ ) due to the characteristic large $d$-band separation from the Fermi energy.
\end{abstract}

(C) 2010 American Institute of Physics. [doi:10.1063/1.3511341]

\section{INTRODUCTION}

During ultrashort pulsed laser heating of metals, the low specific heat of electrons leads to a regime in which the electron system temperature can increase by several orders of magnitude, while the lattice or phonon temperature remains effectively unchanged. During this nonequilibrium period, the large electron temperature changes induced by powerful, ultrashort laser pulses can lead to thermal excitations of subconduction band electrons far below the Fermi energy. The electronic scattering rates for metals predicted by free electron theory are no longer valid in this regime and a clear understanding of the full electronic structure is essential. Noble metals all have characteristic flat $d$-bands several electron volts below the Fermi energy. When ultrashort pulsed laser heating is powerful enough to excite these $d$-band electrons, the electron-phonon scattering rates have been shown to increase by an order of magnitude. ${ }^{1-3}$ While this scattering effect has been recognized, the contributions of $d$-band excitations to the electronic, thermal, and thermoelectric properties of metals during this picosecond time scale have not been explicitly addressed.

Large changes in material properties in metals during strong electron-phonon nonequilibrium are due to Fermi smearing of the electron distribution into bands far removed (a few electron volts) from the Fermi energy, creating a large change in electron and hole number density. ${ }^{4}$ These subFermi energy excitations have also been shown to affect ther-

\footnotetext{
${ }^{a)}$ Electronic mail: pehopki@sandia.gov.
}

mal conductivity and melting, ${ }^{5,6}$ thermal boundary conductance, ${ }^{7}$ and optical absorption. ${ }^{8}$ In this work, we investigate the effects of these large electronic thermal excitations on the ultrafast thermoelectric properties of bulk gold. We characterize the results presented in this work as ultrafast properties since they exist under conditions of electronphonon nonequilibrium (i.e., they are relevant in the picosecond temporal regime) and reiterate that the results in this work are only valid in this regime. Under conditions of strong electron-phonon nonequilibrium (relatively high electron temperatures and relatively low lattice temperatures, i.e., $T_{e} \gg T_{L}$, where $T_{L}$ is the lattice temperature), we find that the Wiedemann-Franz law breaks down and the Seebeck coefficient is massively enhanced, leading to a temporary increase in the thermoelectric figure of merit, $Z T$, in bulk gold by several orders of magnitude. This predicted increase in $Z T$ in $\mathrm{Au}$ is achievable without any structural modification or nanostructuring and is based solely on the intrinsic properties of Au. Although we perform calculations for $\mathrm{Au}$, we expect similar results for the other noble metals $(\mathrm{Ag}$ and $\mathrm{Cu})$ due to the characteristic large $d$-band separation from the Fermi energy and subsequent similar trends in the high- $T_{e}$ thermophysical properties among the noble metals.

\section{COMPUTATIONAL DETAILS}

The thermoelectric figure of merit, $Z T$, is given by $Z T$ $=S^{2} \sigma T / \kappa$, where $\sigma$ is the electrical conductivity, $T$ the temperature of the thermal carriers, and $\kappa$ the thermal conduc- 
tivity. To evaluate $Z T$ for any material, $S, \sigma$, and $\kappa$ must be determined. The Seebeck coefficient of a material is given by $^{9}$

$$
S=-\frac{1}{e T} \frac{\int_{\varepsilon}(\varepsilon-\mu) \frac{\partial f}{\partial \varepsilon} D_{\varepsilon} v^{2} \tau d \varepsilon}{\int_{\varepsilon} \frac{\partial f}{\partial \varepsilon} D_{\varepsilon} v^{2} \tau d \varepsilon},
$$

where $e$ is the electron charge, $T$ the temperature, $v$ the carrier velocity, $\tau$ the relaxation time of the electrons, $\varepsilon$ the electron energy, $\mu$ the chemical potential, $f$ the Fermi distribution, and $D_{\varepsilon}$ the electron density of states per unit volume per unit energy. The electrical and thermal conductivities of a metal are given by ${ }^{9}$

$$
\sigma=-\frac{e^{2}}{3} \int_{\varepsilon} \frac{\partial f}{\partial \varepsilon} D_{\varepsilon} v^{2} \tau d \varepsilon
$$

and

$$
\kappa=\frac{1}{3} \int_{\varepsilon}\left(\varepsilon-\varepsilon_{F}\right) \frac{\partial f}{\partial T} D_{\varepsilon} v^{2} \tau d \varepsilon,
$$

respectively, where $\varepsilon_{F}$ is the Fermi energy. Note that, in Eq. (3), $C=\int_{\varepsilon}\left(\varepsilon-\varepsilon_{F}\right)(\partial f / \partial T) D_{\varepsilon} d \varepsilon$, where $C$ is the volumetric heat capacity, so in metals, Eq. (3) reduces to $\kappa=C v \ell / 3$, where $\ell$ is the mean free path, since $v$ and $\ell=v \tau$ are constant on the Fermi surface. Given these constant electron transport properties, then $v^{2}=2 \varepsilon / m^{*}$ where $m^{*}$ is the effective mass of the conducting electrons and we can rewrite Eqs. (1)-(3) for noble metals in the low excitation regime (no $d$-band excitations) as

$$
\begin{aligned}
& S=-\frac{1}{e T}\left[\frac{\int_{\varepsilon} \varepsilon^{2} \frac{\partial f}{\partial \varepsilon} D_{\varepsilon} d \varepsilon}{\int_{\varepsilon} \frac{\partial f}{\partial \varepsilon} D_{\varepsilon} d \varepsilon}-\mu\right], \\
& \sigma=-\frac{2 e^{2} \tau}{3 m^{*}} \int_{\varepsilon} \varepsilon \frac{\partial f}{\partial \varepsilon} D_{\varepsilon} d \varepsilon, \\
& \kappa=\frac{2 \tau}{3 m^{*}} \int_{\varepsilon}\left(\varepsilon-\varepsilon_{F}\right) \varepsilon \frac{\partial f}{\partial T} D_{\varepsilon} d \varepsilon .
\end{aligned}
$$

Note that, in noble metals in the low excitation regime, the electrons participating in transport can be assumed to have the free electron mass, $m$. In addition, the electron relaxation time on the Fermi surface, which is dominated by electronelectron and electron-phonon scattering, is independent of electron energy. For example, in $\mathrm{Au}$, the free electron scattering time is only dependent on temperature, ${ }^{10}$ and is estimated by ${ }^{11,12} \tau^{-1} \approx A T^{2}+B T$, where $A$ and $B$ are coefficients related to the electron-electron and electron-phonon scattering processes, respectively. ${ }^{10}$

Subjected to low energy inducing low temperature excitations (i.e., energies or temperatures that do not drastically change the number density around the Fermi level), ${ }^{2,4,7,13}$ the total density of states in metals can be approximated by the conduction band density of states at the Fermi energy. In this regime, the chemical potential is approximately equal to the Fermi energy, so Eqs. (4)-(6) reduce to ${ }^{9}$

$$
\begin{aligned}
& S=-\frac{\pi^{2} k_{B}^{2} T}{2 e \varepsilon_{F}}, \\
& \sigma=\frac{n e^{2} \tau}{m}, \\
& \kappa=\frac{\pi^{2} n k_{B}^{2} T \tau}{3 m} .
\end{aligned}
$$

where $k_{B}$ is the Boltzmann's constant and $n$ is the conduction band number density. This leads to the well known expressions for the Lorentz number, $L=\pi^{2} k_{B}^{2} /\left(3 e^{2}\right)$, and the thermoelectric figure of merit of a metal, $Z T=S^{2} / L$ $=3 \pi^{2} k_{B}^{2} T^{2} /\left(4 \varepsilon_{F}^{2}\right)$. The constant Lorentz number for a metal under these low excitation conditions is the basis of the Wiedemann-Franz Law. ${ }^{14}$ The Seebeck coefficient [Eq. (7)] leads to an extremely small value for $Z T$ in a metal under these conditions. At room temperature $Z T=0.000162$ for $\mathrm{Au}$, which is nearly three orders of magnitude lower than the value for common semiconductor structures used in thermoelectric applications $\left(Z T \sim 1\right.$ for $\mathrm{Bi}_{2} \mathrm{Te}_{3}$ alloys at room temperature). ${ }^{15,16}$

After short pulsed laser excitations creating electronphonon nonequilibrium, the thermalized electron system can achieve electron temperatures, $T_{e}$, upwards of $10,000 \mathrm{~K}$ before coupling with and losing energy to the lattice. ${ }^{2,5,6}$ This results in substantial smearing of the electron distribution into the underlying electron orbitals. In this case, the density of states of the excited electrons participating in transport cannot be approximated by the conduction band density alone but rather the density of the subconduction band orbitals, such as the $d$-bands in noble metals, must be included. In the calculations discussed in this work, we use an $a b$ initio density of states for Au calculated with the approach discussed previously. ${ }^{17}$ In short, the density of states of gold was calculated using a plane wave pseudopotential density functional approach ${ }^{18}$ as implemented in the code QUANTUM ESPRESSO. ${ }^{19,20}$ The Perdew-Zunger form for the local density approximation was assumed for exchange and correlation effects in the metal. ${ }^{21}$ Gold atoms were described using an ultrasoft pseudopotential. ${ }^{22}$ A plane wave cutoff of $70 \mathrm{Ry}$ was used in all calculations and a Monkhorst Pack k-point $\operatorname{mesh}^{23}$ of $48 \times 48 \times 48$ was used for integrations in the first Brillouin zone. The tetrahedron approach was used to determine the density of states based on a fine k-grid in the Brillouin zone. ${ }^{24}$ The chemical potential of Au at these high electron temperatures was calculated using the procedure discussed by Lin et al., ${ }^{2}$ where the chemical potential at a given electron temperature is adjusted to match the electronic number density of the conduction band and first-subFermi level $d$-orbital $\left(5 d^{10}\right)$.

As we are interested in determining the thermoelectric properties of $\mathrm{Au}$ as a function of temperature, we must consider a temperature range in which the change in the electronic and phononic structure of $\mathrm{Au}$ is static, as changes in 


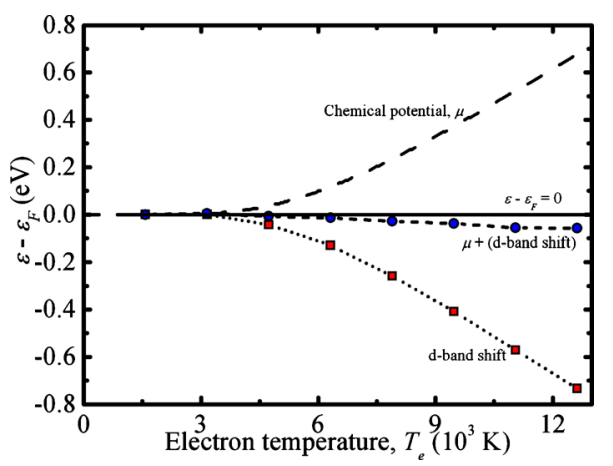

FIG. 1. (Color online) Calculated shift in the $5 d^{10}$ band density of states and the chemical potential as a function of temperature. Also shown is the difference between the chemical potential and the $d$-band shift $[\mu$ $+(d$-band shift $)]$. The shift in the $d$-band density of states is minimal up to electron temperatures of $\sim 10000 \mathrm{~K}$, after which the $d$-band begins to migrate to lower energies, away from the chemical potential. At 10,000 K, the energy shift in the $d$-band is less than $5 \%$, but this increases with higher electron temperatures.

the electronic and phononic density of states could change scattering times and carrier velocities. Recoules et $a l^{25}$ showed that at energies of $6 \mathrm{eV}$, Au vibrational properties showed a strong increase in amplitude and $d$-band-chemical potential separation increases. This is due to the fact that the excitation of $5 d^{10}$ electrons reduces electron screening and makes the effective electron-ion potential more attractive, resulting in more localized $5 d^{10}$ states. ${ }^{25}$ This causes shrinkage in the width of the $d$-bands causing the edge of the $5 d^{10}$ band to decrease in energy more than the corresponding increase in chemical potential. To ensure that we are in a range in which the $d$-band does not shift with respect to the chemical potential, we use finite temperature density functional theory ${ }^{26}$ to self-consistently calculate the Au density of states at several different electron temperatures based on the Fermi-Dirac distribution. Under the assumption that the excited electrons are out of equilibrium with the lattice, the lattice constant is kept fixed at the equilibrium value for all calculations. We plot the calculated shift in the $d$-band and the chemical potential as a function of temperature in Fig. 1. Also shown is the difference between the chemical potential and the $d$-band shift $[\mu+(d$-band shift $)]$. The shift in the $d$-band density of states is minimal up to electron temperatures of $\sim 10,000 \mathrm{~K}$, at which point the $d$-band begins to migrate to lower energies with respect to the chemical potential. At $10,000 \mathrm{~K}$, the energy shift in the $d$-band is less than $5 \%$. Therefore, we restrict calculations in this work to electron temperatures less than $10,000 \mathrm{~K}$ to minimize the effects of changes in the $5 d^{10}$ band density of states.

To determine the thermoelectric figure of merit of a metal under large electron-phonon nonequilibrium we consider $S^{2}$ and $L$ for various electron temperatures assuming a lattice at $300 \mathrm{~K}$. In this case, however, since the effective masses in the $d$-bands of the metal are much higher than those in the $s / p$-bands, we cannot make the simplifying assumptions made to derive Eqs. (4)-(6) (i.e., a constant effective mass). Therefore, we replace the product of the density of states, velocity, and scattering time in Eqs. (1)-(3) with $\left(D_{\varepsilon, s p} v_{s p}^{2} \tau_{s p}+D_{\varepsilon, d} v_{d}^{2} \tau_{d}\right)$, to consider the electrons in the different bands traveling at different velocities and subjected to different scattering rates. With this separation of the $s / p$ and $d$-band density of states, the expression for Seebeck coefficient and Lorentz number becomes

$$
S=-\frac{1}{e T_{e}} \frac{\int_{\varepsilon}(\varepsilon-\mu) \frac{\partial f}{\partial \varepsilon}\left(D_{\varepsilon, s p} v_{s p}^{2} \tau_{s p}+D_{\varepsilon, d} v_{d}^{2} \tau_{d}\right) d \varepsilon}{\int_{\varepsilon} \frac{\partial f}{\partial \varepsilon}\left(D_{\varepsilon, s p} v_{s p}^{2} \tau_{s p}+D_{\varepsilon, d} v_{d}^{2} \tau_{d}\right) d \varepsilon}
$$

and

$$
L=-\frac{1}{e^{2} T_{e}} \frac{\int_{\varepsilon}\left(\varepsilon-\varepsilon_{F}\right) \frac{\partial f}{\partial T_{e}}\left(D_{\varepsilon, s p} v_{s p}^{2} \tau_{s p}+D_{\varepsilon, d} v_{d}^{2} \tau_{d}\right) d \varepsilon}{\int_{\varepsilon} \frac{\partial f}{\partial \varepsilon}\left(D_{\varepsilon, s p} v_{s p}^{2} \tau_{s p}+D_{\varepsilon, d} v_{d}^{2} \tau_{d}\right) d \varepsilon},
$$

respectively. Since the thermoelectric phenomena examined in this study occurs before electron-phonon equilibration, we assume that the electrons are traveling nearly ballistically, and, therefore, are represented by their Fermi velocities. ${ }^{27}$ The Fermi velocity in the $s$ - and $p$-bands of $\mathrm{Au}$ is 1.4 $\times 10^{6} \mathrm{~m} \mathrm{~s}^{-1}{ }^{28}$ For the $d$-band electrons, the Fermi velocity is decreased due to the increased band width compared to the $s / p$-band ${ }^{29}$ and can be estimated as $40 \%$ of the $s / p$-band Fermi velocity for $\mathrm{Au}^{29-31}$

Note that in Eqs. (10) and (11), $\tau$ can no longer be factored out of the integral as in Eqs. (4)-(6). The scattering times are band-dependent due to the differing effective masses of electrons in the $s / p$-and $d$-bands. ${ }^{32}$ The form of the scattering time in each band is given by $\tau_{s p / d}=\left(A_{s p / d} T_{e}^{2}\right.$ $\left.+B_{s p / d} T_{L}\right)^{-1}$, where we assume a lattice temperature of $T_{L}$ $=300 \mathrm{~K}$ since our goal is to study the thermoelectric properties of Au under conditions of strong electron-phonon nonequilibrium. While the scattering coefficients $(A$ and $B)$ are usually assumed to be constants, at high electron temperatures, there will be a change in the electronic number density in the $s / p$ - and $d$-bands and hence a change in the electronic scattering rates. Given that the scattering rates are related to the electronic number density, ${ }^{10}$ we describe the scattering times as

$$
\tau_{s p}=\left[A_{s p}^{0}\left(1+n_{d}\right) T_{e}^{2}+B_{s p}^{0}\left(1+n_{d}\right) T_{L}\right]^{-1}
$$

and

$$
\tau_{d}=\left[A_{d}^{0}\left(1-n_{d}\right) T_{e}^{2}+300 B_{d}^{0}\left(1-n_{d}\right)\right]^{-1},
$$

where $n_{d}$ is the number of electrons excited from below the $d$-band edge in Au into the $s / p$-band and is temperature dependent. We determine $n_{d}$ by integrating the product of the total density of states below the $d$-band edge and the number of available states as a function of energy; i.e., $n_{d}=\int D_{\varepsilon, d}(1$ $-f) d \varepsilon$. Note that the changes in band number density yield an increase in scattering rates in the $s / p$-band but a decrease in scattering rates in the $d$-band since the number density is increasing in the $s / p$-band while decreasing in the $d$-band. Equation (12) has a similar form of the electron-electron scattering rate as the phenomenological expression presented by Chan et al. ${ }^{6}$ However, since we assume an equilibrated electron system, we do not include any weighting factor to 
account for heavy holes in the $s / p$-band. Although we take the electrons and holes as equilibrated in this work, we still assume that the electrons have not coupled with the phonons so the phonon system can still be treated as an equilibrated system at $300 \mathrm{~K}$, separate from the high temperature, equilibrated electronic system. This is a valid assumption since the electron-hole relaxation time in $\mathrm{Au}$ is nearly an order of magnitude faster than the electron-phonon equilibration time. ${ }^{8}$ In addition, we account for the change in the electronphonon scattering rates due to the change in number density in the bands in Eq. (13), since the strength of the electronphonon interaction and subsequent energy transfer rate will increase (decrease) as the number of electrons increases (decreases). ${ }^{2,10,32,33}$ For calculations of Eqs. (12) and (13), we turn to the procedure outlined by Chan et al., ${ }^{6}$ that is, we treat the electronic scattering in the $s / p$-band similar to that observed from low temperature $\mathrm{Au}$ resistivity measurements and that in the $d$-band similar to that from low temperature Ni resistivity measurements. ${ }^{11,34}$ Therefore, $A_{s p}^{0}=1.2 \times 10^{7} \mathrm{~K}^{-2} \mathrm{~s}^{-1}, \quad B_{s p}^{0}=1.23 \times 10^{11} \mathrm{~K}^{-1} \mathrm{~s}^{-1}, \quad A_{d}^{0}=1.4$ $\times 10^{6} \mathrm{~K}^{-2} \mathrm{~s}^{-1}$, and $B_{d}^{0}=1.624 \times 10^{13} \mathrm{~K}^{-1} \mathrm{~s}^{-1}$.

\section{DISCUSSION}

Figures 2(a)-2(c) show $S^{2}, L$, and $Z T$, respectively, for $\mathrm{Au}$ as a function of electron temperature normalized by their respective values at $T_{e}=300 \mathrm{~K}$ (i.e., electron-phonon equilibrium). It is interesting to note that the square of the Seebeck coefficient [Fig. 2(a)] increases over 4 orders of magnitude at electron temperatures of $10,000 \mathrm{~K}$. This is consistent with the conceptual definition of the Seebeck coefficient, which represents the average energy of a mobile electron, or the average heat current carried per electron. ${ }^{9}$ The sharp $d$-band edge represents a large spike in average electron energy, and as temperature is increased, the average energy increases corresponding to the increases in $S^{2}$. However, the temperature onset of the increase in $S^{2}$ $(\sim 4000 \mathrm{~K})$ does not correspond exactly to the onset of $d$-band excitations in $\mathrm{Au}(\sim 3000 \mathrm{~K})$ due to electron scattering processes. ${ }^{2}$ The immensely strong scattering processes suppress the effects of the increased electron energy on $S^{2}$ until about $0.1 \mathrm{eV}$ into the $d$-bands; i.e., the scattering term outweighs the energy and density of states increase in Eq. (10) until $\sim 4000 \mathrm{~K}$. The leveling off of $S^{2}$ around $10,000 \mathrm{~K}$ is ascribed to a decrease in the change of electron energy with electronic excitations at energies beyond the initial $d$-band peak.

The normalized Lorentz number [Fig. 2(b)] is a constant up to the onset of $d$-band thermal excitation after which point $L$ decreases with a local minimum at $\sim 7000 \mathrm{~K}$. Since $L$ $=\kappa /(\sigma T)$, the increase in $L$ due to electron number density is greater than the increase in $L$ due to thermal energy as the $d$-bands become excited. This arises due to the large rise in electron population at the $d$-band edge. At low temperatures and small perturbations, the density of states around the Fermi energy of a free electron metal is approximately constant leading to the constant Lorentz number. ${ }^{35}$ However, with the thermal excitations from the $d$-band, the density of states in Au becomes highly nonlinear, causing a decrease in
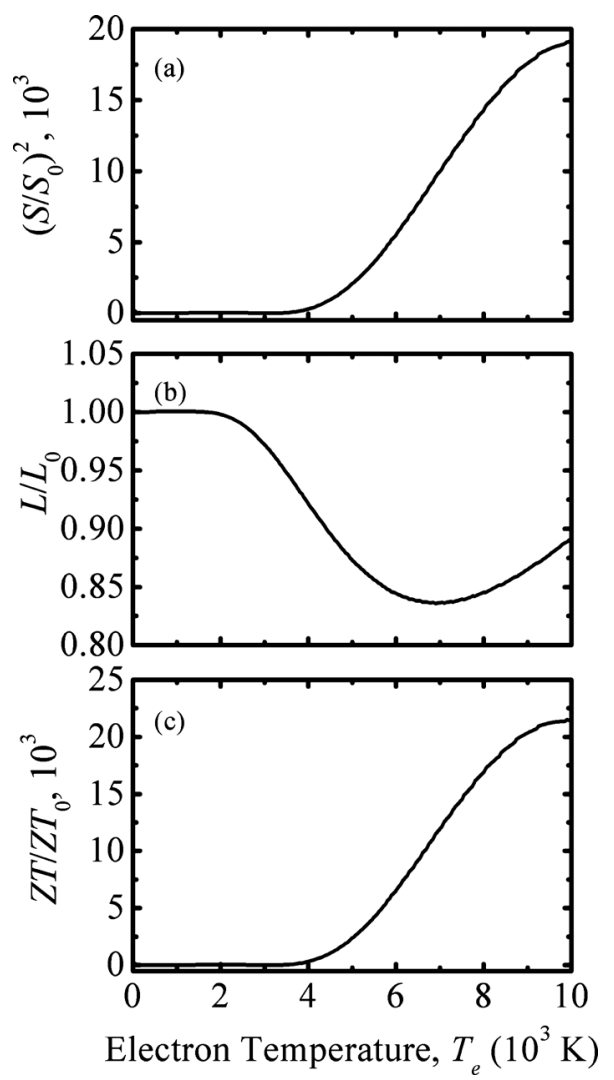

FIG. 2. (a) Square of the Seebeck coefficient normalized to $S^{2}$ at $T$ $=300 \mathrm{~K}$ showing a maximum increase in over 4 orders of magnitude at electron temperatures above $\sim 7000 \mathrm{~K}$. (b) Normalized Lorentz number as a function of temperature which is a constant up to the onset of $d$-band thermal excitation. The dependency of $L$ on temperature indicates that upon $d$-band excitation, the Wiedemann-Franz Law no longer applies. (c) Normalized thermoelectric figure of merit for Au under conditions of strong electron-phonon nonequilibrium as a function of electron temperature. $Z T$ massively increases with the onset of $d$-band thermal excitations with a predicted maximum increase in $Z T$ of 22,000 times that at room temperature during electron-phonon equilibrium conditions. We reiterate that the results in this work are only valid in this regime of strong electron-phonon nonequilibrium.

$L$. This shows that the Wiedemann-Franz Law no longer holds for noble metals under strong electron-phonon nonequilibrium in which $d$-band electrons are thermally activated.

Figure 2(c) shows the normalized thermoelectric figure of merit for $\mathrm{Au}$ under conditions of strong electron-phonon nonequilibrium as a function of electron temperature. The figure of merit in $\mathrm{Au}$ dramatically increases with the onset of $d$-band thermal excitations with a predicted maximum increase of $\sim 22000$ times that at room temperature during electron-phonon equilibrium conditions. We note, as previously mentioned, that all the properties calculated in this work are considered "ultrafast" properties as they exist under conditions of strong electron-phonon nonequilibrium, which, after short pulsed laser heating, only persists for time scales on the order of picoseconds in metals.

To put this increase into perspective, the highest increase in $Z T$ reported was measured in $\mathrm{Bi}_{2} \mathrm{Te}_{3} / \mathrm{Sb}_{2} \mathrm{Te}_{3}$ superlattices $(Z T \sim 2.5)$ that showed a factor of 2.5 increase over bulk $\mathrm{Bi}_{2-\mathrm{x}} \mathrm{Sb}_{\mathrm{x}} \mathrm{Te}_{3}(Z T \sim 1.0) .^{36}$ The highest theoretical increase in $Z T$ was predicted in low dimensional $\mathrm{Bi}_{2} \mathrm{Te}_{3}$-like materials, 
where the limiting dimension in a quantum well (twodimensional) or quantum wire (one-dimensional) was 5.0 $\mathrm{nm} .{ }^{37}$ These $Z T$ values were predicted as 5.0 for the quantum well and 14.0 for the quantum wire and the theoretical $Z T$ decreased with increases in the limiting dimension. In bulk gold under strong nonequilibrium conditions, a maximum instantaneous $Z T$ of 3.5 at $10,000 \mathrm{~K}$ is predicted from the model presented in this work, assuming a room temperature equilibrium lattice, and a value of $Z T$ for bulk $\mathrm{Au}$ of 0.000162 . This increase in $Z T$ in $\mathrm{Au}$ is achievable without any structural modification or nanostructuring and is based solely on the intrinsic properties of bulk Au. Although there may be some degree of melting after the electron system at $10,000 \mathrm{~K}$ has equilibrated with the phonon system, the ultrafast properties studied in this work will be unaffected by this since we study these properties in Au before electronphonon equilibration begins (i.e., before the electron loses energy to the lattice causing any structural or phase change).

Although we predict a massive enhancement in $Z T$ during electron-phonon nonequilibrium, the thermoelectric benefit may not be available for traditional thermoelectric applications. For example, these calculations were performed assuming a room temperature lattice. This means that the time in which the metal can be used as an efficient thermoelectric under these conditions is limited to the electronphonon equilibration time after short pulsed laser excitation, that is, about 5 ps. $^{2}$ A state-of-the-art short pulsed laser system with $1 \mathrm{GHz}$ repetition rate yields a usable duty cycle to extract the thermoelectric benefit from the excited metal of $0.5 \%$. Using the nonequilibrium and equilibrium $Z T$ of $\mathrm{Au}$ and accounting for this duty cycle yields a best case average $Z T$ of 0.018 for a gold target irradiated with short pulses. It remains to be seen whether it is possible to design a system that can utilize the ultrafast thermoelectric properties of metals during electron-phonon nonequilibrium in applications involving ultrashort, high-power thermal fluxes. However, it should be possible to measure the average enhancement in the metal $Z T$ value using a short pulsed laser system to verify the analysis presented here. The breakdown of the Wiedemann-Franz relation could also have important implications in experimental situations exhibiting conditions of strong electron-phonon nonequilibrium (laser pulses, shock wave physics), where electrical conductivity measurements can be used to estimate thermal conductivity. Using the equilibrium Lorentz constant in these scenarios will lead to an overestimate of the actual thermal conductivity.

\section{CONCLUSIONS}

In summary, we investigated the effects of large electronic thermal excitations on the thermoelectric transport properties of gold. Under conditions of strong electronphonon nonequilibrium, we show a massive enhancement in the Seebeck coefficient and find that the Wiedemann-Franz law breaks down. Although we perform representative calculations for $\mathrm{Au}$, these results are similar for the other noble metals $(\mathrm{Ag}$ and $\mathrm{Cu})$ due to the characteristic large $d$-band separation from the Fermi energy.

\section{ACKNOWLEDGMENTS}

P.E.H. is greatly appreciative for funding from the LDRD Program Office through the Harry S. Truman Fellowship Program. J.C.D. and T.S.E. are grateful for financial support from the National Science Foundation through the Graduate Research Fellowship Program. Sandia National Laboratories is a multiprogram laboratory operated by Sandia Corporation, a wholly owned subsidiary of LockheedMartin Corporation, for the United States Department of Energy's National Nuclear Security Administration under Contract DE-AC04-94AL85000. First principle calculations were performed on the Intel Cluster at the Cornell Nanoscale Facility, which is part of the National Nanotechnology Infrastructure Network funded by the National Science Foundation.

${ }^{1}$ Z. Lin and L. V. Zhigilei, Proc. SPIE 6261, 62610U (2006).

${ }^{2}$ Z. Lin, L. V. Zhigilei, and V. Celli, Phys. Rev. B 77, 075133 (2008).

${ }^{3}$ Z. Lin and L. V. Zhigilei, Appl. Surf. Sci. 253, 6295 (2007).

${ }^{4}$ P. E. Hopkins, J. Heat Transfer 132, 014504 (2010).

${ }^{5}$ W.-L. Chan, R. S. Averback, D. G. Cahill, and Y. Ashkenazy, Phys. Rev. Lett. 102, 095701 (2009).

${ }^{6}$ W.-L. Chan, R. S. Averback, D. G. Cahill, and A. Lagoutchev, Phys. Rev. B 78, 214107 (2008).

${ }^{7}$ P. E. Hopkins, T. E. Beechem, J. C. Duda, J. L. Smoyer, and P. M. Norris, Appl. Phys. Lett. 96, 011907 (2010).

${ }^{8}$ W.-L. Chan, R. S. Averback, and D. G. Cahill, Appl. Phys. A: Mater. Sci. Process. 97, 287 (2009).

${ }^{9}$ G. Chen, Nanoscale Energy Transport and Conversion: A Parallel Treatment of Electrons, Molecules, Phonons, and Photons (Oxford University Press, New York, 2005).

${ }^{10}$ M. Kaveh and N. Wiser, Adv. Phys. 33, 257 (1984).

${ }^{11}$ A. H. MacDonald, Phys. Rev. Lett. 44, 489 (1980).

${ }^{12}$ X. Y. Wang, D. M. Riffe, Y.-S. Lee, and M. C. Downer, Phys. Rev. B 50, 8016 (1994).

${ }^{13}$ P. E. Hopkins, Appl. Phys. Lett. 96, 041901 (2010).

${ }^{14}$ R. Franz and G. Wiedemann, Ann. Phys. 165, 497 (1853).

${ }^{15}$ H. G. Goldsmid, Thermoelectric Refrigeration (Plenum, New York, 1964).

${ }^{16}$ G. Chen, M. Dresselhaus, G. Dresselhaus, J. P. Fleurial, and T. Caillat, Int. Mater. Rev. 48, 45 (2003).

${ }^{17}$ P. E. Hopkins and D. A. Stewart, J. Appl. Phys. 106, 053512 (2009).

${ }^{18}$ R. M. Martin, Electronic Structure: Basic Theory and Methods (Cambridge University Press, New York, 2004).

${ }^{19}$ S. Scandolo, P. Giannozzi, C. Cavazzoni, A. Pasquarello, and S. Baroni, Z. Kristallogr. 220, 574 (2005).

${ }^{20}$ P. Giannozzi, S. Baroni, N. Bonini, M. Calandra, R. Car, C. Cavazzoni, D. Ceresoli, G. L. Chiarotti, M. Cococcioni, I. Dabo, A. Dal Corso, S. de Gironcoli, S. Fabris, G. Fratesi, R. Gebauer, U. Gerstmann, C. Gougoussis, A. Kokalj, M. Lazzeri, L. Martin-Samos, N. Marzari, F. Mauri, R. Mazzarello, S. Paolini, A. Pasquarello, L. Paulatto, C. Sbraccia, S. Scandolo, G. Sclauzero, A. P. Seitsonen, A. Smogunov, P. Umari, and R. M. Wentzcovitch, J. Phys.: Condens. Matter 21, 395502 (2009).

${ }^{21}$ J. P. Perdew and A. Zunger, Phys. Rev. B 23, 5048 (1981).

${ }^{22} \mathrm{We}$ used the Au.pz-d-rrkjus.UPF pseudopotential from the http:// www.quantum-espresso.org distribution.

${ }^{23}$ H. J. Monkhorst and J. D. Pack, Phys. Rev. B 13, 5188 (1976).

${ }^{24}$ P. E. Blöchl, O. Jepsen, and O. K. Andersen, Phys. Rev. B 49, 16223 (1994).

${ }^{25}$ V. Recoules, J. Clerouin, G. Zerah, P. M. Anglade, and S. Mazevet, Phys. Rev. Lett. 96, 055503 (2006).

${ }^{26}$ N. D. Mermin, Phys. Rev. 137, A1441 (1965).

${ }^{27}$ T. Saito, O. Matsuda, and O. B. Wright, Phys. Rev. B 67, 205421 (2003).

${ }^{28}$ N. W. Ashcroft and N. D. Mermin, Solid State Physics (Saunders College, Fort Worth, 1976).

${ }^{29}$ W. A. Harrison, Electronic Structure and the Properties of Solids: The Physics of the Chemical Bond (W. H. Freeman, San Francisco, 1980).

${ }^{30}$ E. Heiner, Phys. Status Solidi B 148, 599 (1988).

${ }^{31}$ R. Knorren, K. H. Bennemann, R. Burgermeister, and M. Aeschlimann, Phys. Rev. B 61, 9427 (2000).

${ }^{32}$ J. M. Ziman, Electrons and Phonons (Clarendon, Oxford, 1960). 
${ }^{33}$ M. I. Kaganov, I. M. Lifshitz, and L. V. Tanatarov, Sov. Phys. JETP 4, 173 (1957).

${ }^{34}$ D. S. Ivanov and L. V. Zhigilei, Phys. Rev. B 68, 064114 (2003).

${ }^{35}$ C. Kittel, Introduction to Solid State Physics (Wiley, New York, 1996).

${ }^{36}$ R. Venkatasubramanian, E. Siivola, T. Colpittas, and B. O'Quinn, Nature
(London) 413, 597 (2001).

${ }^{37}$ M. S. Dresselhaus, G. Dresselhaus, X. Sun, Z. Zhang, S. B. Cronin, T. Koga, J. Y. Ying, and G. Chen, Nanoscale Microscale Thermophys. Eng. 3, 89 (1999). 